\title{
电磁驱动器与矩形静压空气止推轴承混合支承 工作台的设计与实现
}

\author{
毛军红 ${ }^{\oplus}$, 李黎川 ${ }^{\circledR}$
}

西安交通大学(1)机械工程学院; (2)电气工程学院, 西安 710049

E-mail: jhmao@mail.xjtu.edu.cn

2008-01-05 收稿, 2008-08-05 接受

国家自然科学基金(批准号: 50475083)资助项目

摘要 研究了电磁驱动器与矩形静压空气止推轴承混合支承工作台的设计与实现. 由于工作台 的单一平面静压空气止推轴承同时采用电磁驱动器构成闭环主动控制, 具有结构简单精度高的 特点. 工作台机械结构对称, 只有一个移动部件, 静压空气止推轴承气浮块与移动台刚性连接, 直 接加工在移动台轴承面上的铁心和 4 个独立线圈构成电磁驱动器. 设计实现了工作台实验原 型, 推导了工作台止推轴承 3 个自由度解耦的模型. 在 DOS 下用 C 语言实现了工作台的闭环控制算 关键词 精密工作台 静压空气轴承 电磁驱动器 主动控制 法, 控制器设计采用任意极点配置, 被控对象的模型忽略了静压空气轴承特性. 工作台在垂直 方向上能实现精密微定位, 并能作为移动台平面 3 个自由度运动的支承, 对平面内运动的行程没 有限制. 工作台定位精度不再取决于空气轴承的设计和加工精度, 无需被动预载.

近年来, 随着超精密部件在计算机、光学和通信 领域应用的日益广泛, 其需求一直快速增长. 由于静 压空气轴承具有高精度和几乎无摩擦的特性, 在超 精密加工领域受到越来越多的关注, 并已经应用于 许多要求纳米级精度机床设备平动和回转运动的实 现. 矩形静压空气止推轴承通过平面气膜将移动台 与支承部件分开, 是一种实现平面超精密工作台简

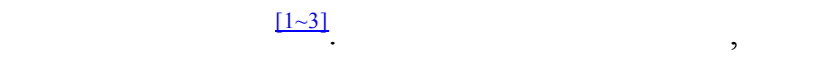
需要采用被动的磁力或真空预载以获得适当的气膜 厚度和好的刚度 ${ }^{[3,4]}$. 使用矩形静压空气止推轴承的 工作台, 具有 6 个自由度运动, 在结构上比采用叠层 的串联结构或并联结构大为简化, 消除了平动关于 重心的对准误差, 平衡质量对底座的力, 以及各轴之 间误差耦合等, 部件几何误差以及运动质量对运动 精度的影响大大减小. 移动台平面的 3 个坐标运动方 向可以采用一个 $X Y \theta_{Z}$ 平面三轴电机驱动的闭环主动 控制, 实现单级(长行程)纳米级运动精度 ${ }^{[3]}$. 矩形静 压空气止推轴承的 3 个自由度是被动的, 其性能取决 于矩形静压空气止推轴承的设计和加工精度.
由于平台止推磁轴承的 3 个自由度都是主动控 制的, 所以磁轴承在实现超精密非接触工作台方面 具有一些突出的优点, 已有应用磁轴承技术实现多 轴超精密工作台的相关研究 ${ }^{[5]}$. 基本上, 磁轴承工作 台可分为电动力型和电磁力型 ${ }^{[6]}$. 前者的磁悬浮力是 线性洛仑兹力, 具有良好的闭环控制性能, 但是需要 使用永磁体, 并且功耗大. Kim和Trumper ${ }^{[7]}$ 做过一个 相关的研究, 其优点是在工作台的上方没有为电磁 铁产生吸力的导磁体或产生预载的永久磁体, 移动 台无热损耗减小了由于热膨胀引起的变形, 方便地 把定子产生的热量传导到基座. 后者尽管磁悬浮是 开环不稳定的, 但是也可以达到具有一定闭环带宽 的纳米级运动精度 ${ }^{[8]}$. 这类磁轴承工作台不需永磁体, 并且极大地减小了功率消耗.

文献[9]研究了一个电磁驱动器和空气轴承混合 支承 3 自由度微动工作台. 在移动台上, 安装了 3 个 止推轴承, 均匀布置在半径 $58 \mathrm{~mm}$ 的圆周上, 实现了 没有机械接触工作台. 每一个止推轴承由电磁驱动 器以及小孔节流空气轴承组成. 驱动器的中心安装 
了一个电容式位移传感器, 驱动器线圈绕在传感器 保持架上, 在止推轴承的导向面上, 围绕驱动器的线 圈有 4 个节流孔. 这类工作台应用中最大的不足是移 动台须为圆形或三角形，而不是通常采用的能够保 证几何和动态对称性的矩形. 此外, 这种工作台结构 复杂, 难于实现. 因为这种工作台的力学特性取决于 空气轴承, 静压气体非线性力学特性使得控制器设 计和控制性能提高存在一定的困难.

本文的研究目的是开发一个主动空气轴承工作 台, 工作台由移动台和基座构成, 移动台的轴承面上 安装了 1 个静压气浮块以及 4 个独立线圈. 静压空气 轴承作为平面支承, 用于承载重力方向的力, 线圈则 构成电磁驱动器. 主动空气轴承能够支承矩形移动 台在平面内 3 个自由度的单级长行程运动, 同时不需 任何辅助驱动器, 就能够对移动台姿态进行微调, 实 现工作台对准调整、姿态校准、以及高度调整等. 此 外, 可以对主动空气轴承工作台的刚度和悬浮高度 进行主动控制, 无需被动预载. 工作台垂直方向的动 态性能取决于空气轴承. 电磁驱动器提供移动台平 面 2 个偏转自由度的刚度. 与文献[9]相比, 主动空气 轴承工作台结构更简单, 偏转自由度的控制特性更 有效.

主动空气轴承工作台只有 1 个移动部件, 实现了 没有传动机构的直接驱动, 这样工作台在 6 个自由度 方向的运动性能都是一致的, 而且保留了矩形静压 空气止推轴承工作台和磁轴承工作台容易加工和动 力学运动学模型简单等特点. 这意味着系统的性能 取决于其伺服控制, 将来可以通过高精度伺服设备 不断提高. 如果 6 自由度反馈控制具有一个隔离的测 量基准, 移动台将完全与基座隔离, 这使得工作台对 干扰的敏感性比被动静压空气轴承工作台小 $10^{3} \sim 10^{4}$ 倍 ${ }^{[5]}$. 通过采用静压空气轴承, 主动空气轴承工作台 开环稳定, 具有功耗低和鲁棒性好的优点. 主动空气 轴承工作台不需要在长行程系统上再叠加一个微细 调整的短行程系统, 是一种实现单级(长行程)纳米级 运动精度工作台的方法.

\section{1 设计分析}

图 1 为主动空气轴承工作台和装配后的移动台 轴承面照片. 气浮块刚性固定在移动台轴承面的中 心, 4 个线圈和 4 个电涡流位移传感器交替均匀布置 在移动台轴承面上, 以减小工作台测量的系统误差,

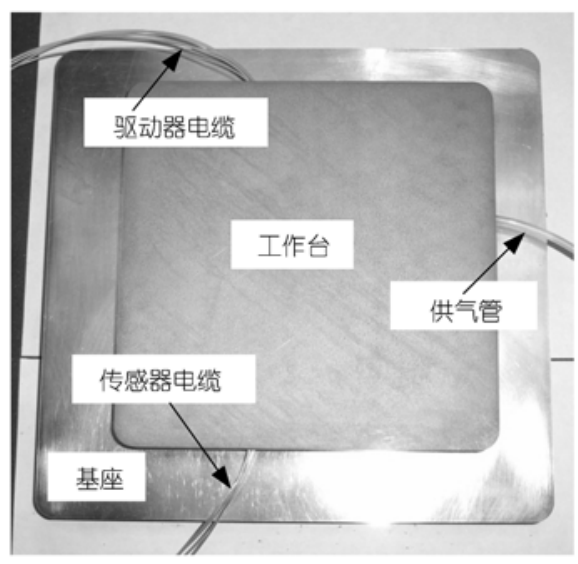

(a)

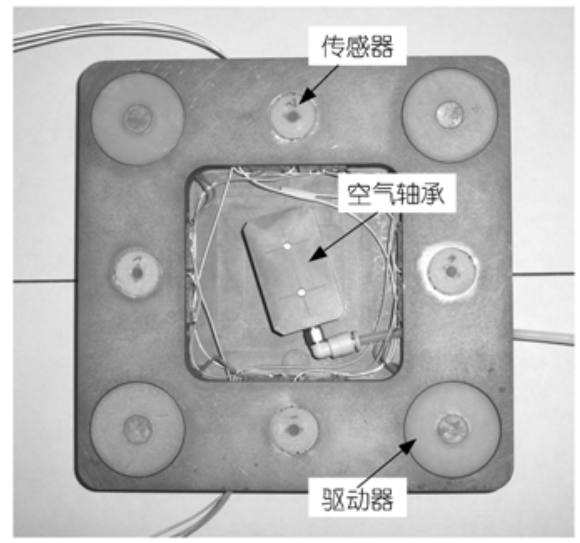

(b)

图 1 主动空气轴承工作台

(a) 工作台; (b) 装配好的移动台轴承面

降低工作台重心. 开环的主动空气轴承工作台为平 面气膜矩形静压空气止推轴承工作台, 因为没有预 载, 工作台垂直方向稳定, 刚度和阻尼特性差. 由于 空气轴承安装在移动台中心位置, 2 个偏转自由度性 能更差. 闭环时, 由于空气轴承的静态工作点由电磁 驱动器闭环控制, 工作台垂直方向上的刚度有显著 提高, 取决于空气轴承的特性, 电磁驱动器提供 2 个 偏转自由度的刚度.

当移动台由空气轴承悬浮起来时, 位移传感器 测量气膜厚度, 并且由非接触电磁驱动器主动控制 气膜厚度. 为了保证矩形移动台几何和动态特性的 对称性, 使用 4 个驱动器和传感器. 刚性移动台使驱 动器的作用力和传感器输出产生耦合. 图 2 为移动台 的驱动器和传感器布局的顶视图及坐标定义, 图 3 为 主动空气轴承工作台系统方框图, 其中 A1 A4 是电 
磁驱动器, 驱动电流分别为 $i_{1} \sim i_{4}, \mathrm{~S} 1 \sim \mathrm{S} 4$ 是电涡流位 移传感器, 其位移增量输出为 $s_{1} \sim s_{4}$. 驱动轴位移 $z, \alpha, \beta$ 与传感器输出 $s_{1} \sim s_{4}$ 的关系为

$$
\left[\begin{array}{l}
z \\
\alpha \\
\beta
\end{array}\right]=f_{1}(s)=\left[\begin{array}{l}
\left(s_{1}+s_{2}+s_{3}+s_{4}\right) / 4 \\
\left(s_{4}-s_{3}\right) / D \\
\left(s_{1}-s_{2}\right) / D
\end{array}\right],
$$

其中 $\boldsymbol{s}=\left[s_{1}, s_{2}, s_{3}, s_{4}\right]^{\mathrm{T}}$.
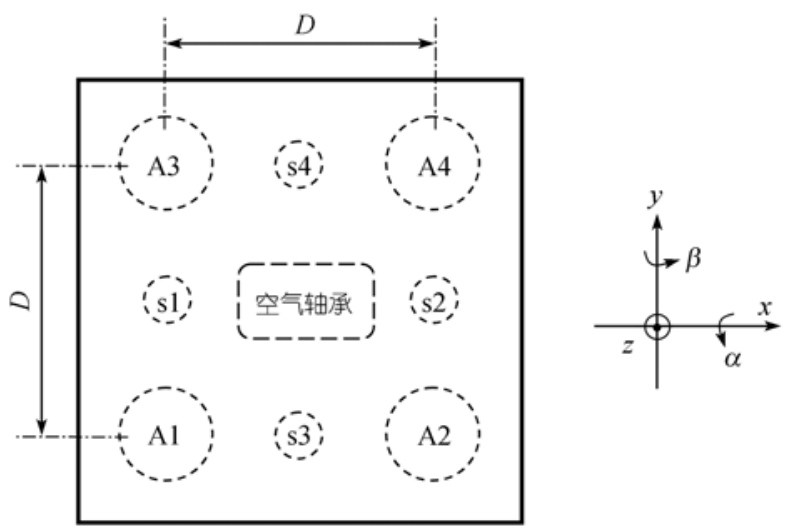

图 2 驱动器和传感器布置

驱动器驱动电流 $i_{1} \sim i_{4}$ 与驱动轴控制电流 $i_{z}, i_{\alpha}, i_{\beta}$ 有如下关系:

$$
\left[\begin{array}{l}
i_{1} \\
i_{2} \\
i_{3} \\
i_{4}
\end{array}\right]=f_{2}(\boldsymbol{i})=\left[\begin{array}{l}
-i_{z}+i_{\alpha}-i_{\beta} \\
-i_{z}+i_{\alpha}+i_{\beta} \\
-i_{z}-i_{\alpha}-i_{\beta} \\
-i_{z}-i_{\alpha}+i_{\beta}
\end{array}\right],
$$

其中 $\boldsymbol{i}=\left[i_{z}, i_{\alpha}, i_{\beta}\right]^{\mathrm{T}}$.

工作台的驱动关于工作台重心对称，消除了各 轴之间的相互影响.

使用电磁驱动器和静压空气悬浮混合支承, 实 现了单一移动部件工作台, 其止推轴承的 3 个自由度 可以进行闭环控制. 静压空气悬浮的优点使得主动 空气轴承工作台开环稳定、鲁棒性好、承载能力高、 发热小、功耗低.

\section{2 实验原型}

为了验证主动空气轴承工作台, 建立了一个实 验原型(图 1)，基座和移动台采用实心软铁，基座的 轴承面磨削加工以保证平面度. 在移动台的轴承面 上, 选用了一个气浮块, 用环氧树脂固定于移动台轴
承面的空腔中心, 空腔比气浮块大, 四周有 8 个直径 $8 \mathrm{~mm}$ 的孔, 将空腔与外部连通. 因此, 空气轴承的 气体可以通过这些孔释放到外部, 静压气膜仅局限 于气浮块. 这种结构能够保护气浮块, 而且静压空气 轴承的性能可由气浮块设计数据计算. 在同一个面 上, 还加工了 4 个环行盲孔用来安装线圈, 和 4 个盲 孔用来安装传感器. 驱动器的工作气隙是移动台轴 承面与铁心顶部的高度差 (图 4). 线圈铜线直径为 $0.47 \mathrm{~mm}$. 电浴流传感器用环氧树脂固定在移动台上, 表 1 为实验原型的机械和力学参数.

在实验原型中, 将 4 个线圈直接安装在移动台上, 构成 4 个独立的有铁芯电磁驱动器. 由于加工和装配 非常简单, 这种驱动器设计非常实用. 同时, 易于保 证驱动器的安装位置精度和工作气隙一致性, 这取 决于加工精度.

为了保持几何对称性, 实验原型具有 4 个独立的 驱动器和位移传感器. 从(1)和(2)式可知, 由这些驱 动器和传感器能够实现止推轴承 3 个自由度的闭环控 制. 此外, 测量噪声有所降低.

\section{3 实验及讨论}

\section{1 主动空气轴承建模}

图 4 为电磁驱动器原理图, 磁极面积为 $A$. 当移 动台处于平衡位置时, 驱动器工作气隙为 $Z_{0}$, 传感器 间隙为 $50 \mu \mathrm{m}$. 驱动器在 $z$ 轴偏离平衡位置的位移增 量表示为 $z_{1} \sim z_{4}$, 单位 $\mathrm{m}$, 在平衡位置时 $z_{1}=$ $z_{2}=z_{3}=z_{4}=0$. 每一个驱动器的吸引力, 以驱动器 1 为例, 方向与 $z$ 轴相反, 由下式计算:

$$
f_{1}=\frac{A B_{1}^{2}}{2 \mu_{0}}=\frac{\mu_{0} A N^{2} I_{0}^{2}}{2\left(Z_{0}+z_{1}\right)} \approx f_{10}-P z_{1}+Q i_{1},
$$

其中

$$
f_{10}=\frac{\mu_{0} A N^{2} I_{0}^{2}}{2 Z_{0}}, \quad P=\frac{\mu_{0} A N^{2} I_{0}^{2}}{Z_{0}^{3}} \text { 和 } Q=\frac{\mu_{0} A N^{2} I_{0}^{2}}{Z_{0}^{2}},
$$

$B_{1}$ 为驱动器线圈产生的磁感应强度 $(\mathrm{T}), I_{0}$ 为线圈偏 置电流(A). 对应于(3)式, 可得其余 3 个驱动器的力 $f_{2}$, $f_{3}$ 和 $f_{4}$, 以及线性化模型, 各个力的参数相同, 并对 应于各自驱动器的位移和驱动电流.

当移动台处于平衡位置时, 移动台中心的平动 和转动位移均为 0 , 即 $z=\alpha=\beta=0$. 假设一定气膜 厚度下空气轴承在垂直方向上提供的力 $F_{\text {air }}$ 为常数, 且与移动台重力和偏置电流产生的力相平衡. 空气 轴承力学特性对于止推轴承 2 个转动轴的影响非常 


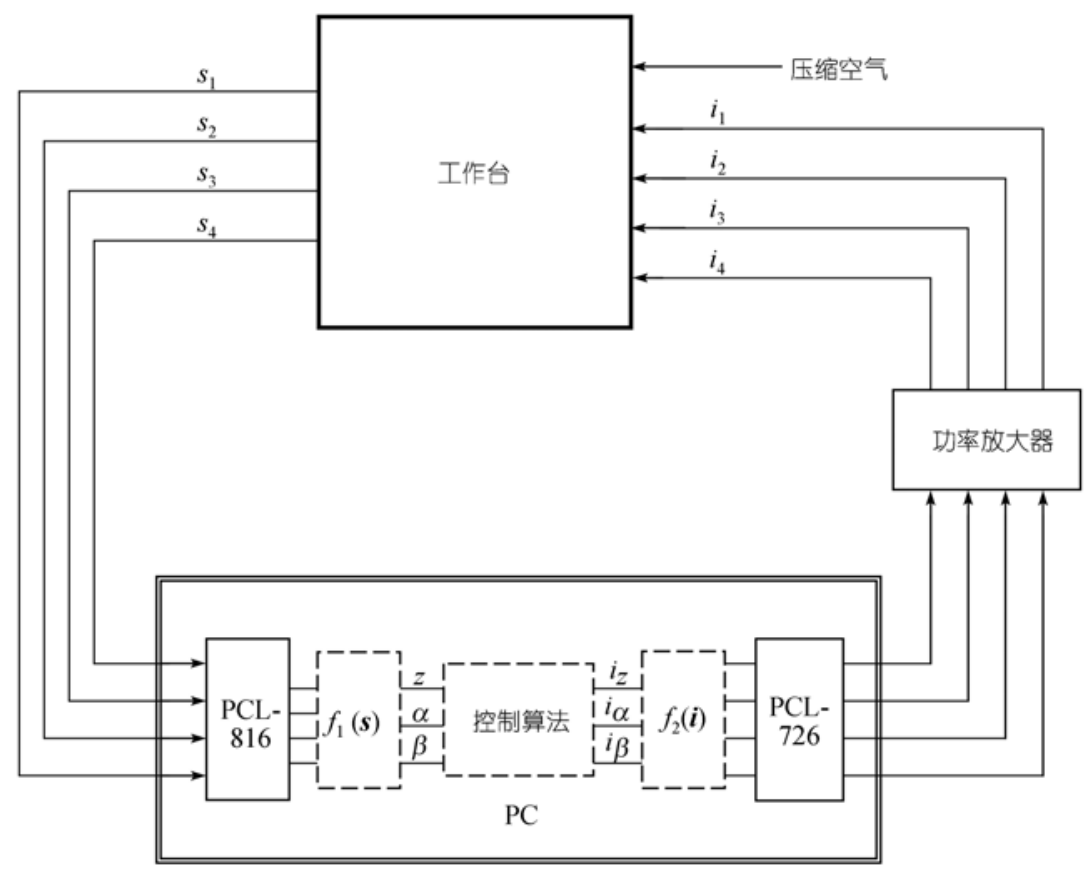

图 3 主动空气轴承工作台系统方框图

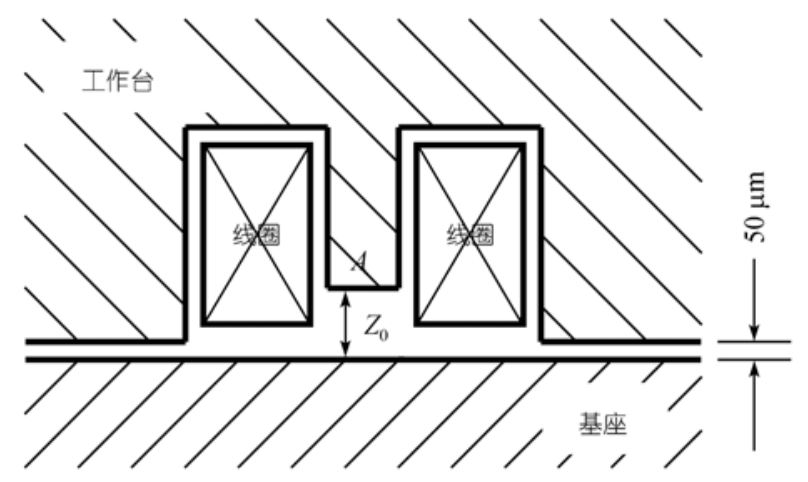

图 4 电磁驱动器原理图

表 1 工作台参数

\begin{tabular}{clc}
\hline & \multicolumn{1}{c}{ 参数 } & 数值 \\
\hline 基座 & 大小 $/ \mathrm{mm} \times \mathrm{mm}$ & $250 \times 240$ \\
& 厚度 $/ \mathrm{mm}$ & 23 \\
\hline 移动台 & 大小 $/ \mathrm{mm} \times \mathrm{mm}$ & $180 \times 180$ \\
& 厚度 $/ \mathrm{mm}$ & 23 \\
& $x$ 或 $y$ 轴转动惯量 $\mathrm{J} / \mathrm{kg} \cdot \mathrm{m}^{2}$ & 0.015 \\
& 驱动器间的距离 $\mathrm{D} / \mathrm{mm}$ & 130 \\
& 质量 $M / \mathrm{kg}$ & 4.8 \\
\hline 驱动器 & 线圈匝数 $N$ & 720 \\
& 线圈电阻 $R / \Omega$ & 6.0 \\
& 工作气隙 $Z_{0} / \mathrm{mm}^{2}$ & 0.9 \\
& 磁极面积 $A / \mathrm{mm}^{2}$ & $1.13 \times 10^{-4}$ \\
\hline 位移传感器 & 测量范围 $/ \mu \mathrm{m}$ & 100 \\
& 输出范围 $/ \mathrm{V}$ & $-10.0 \sim 10.0$ \\
& 灵敏度 $/ \mu \mathrm{m} \cdot \mathrm{V}^{-1}$ & 5 \\
\hline
\end{tabular}

小, 可以忽略不记. 因此, 由(1) (3)式可以得到各驱 动轴解耦的模型, 其中未计及空气轴承力学特性的 影响

$$
\left\{\begin{aligned}
M \ddot{z}= & -M g+F_{\text {air }}-\left(f_{1}+f_{2}+f_{3}+f_{4}\right) \\
= & -M g+F_{\text {air }}-\left(f_{10}+f_{20}+f_{30}+f_{40}\right) \\
& +P\left(z_{1}+z_{2}+z_{3}+z_{4}\right)-Q\left(i_{1}+i_{2}+i_{3}+i_{4}\right) \\
= & 4 P z-4 Q i_{z}, \\
J \ddot{\alpha}= & \frac{D}{2}\left(f_{1}+f_{2}-f_{3}-f_{4}\right) \\
= & \frac{D}{2}\left[P\left(-z_{1}-z_{2}+z_{3}+z_{4}\right)\right. \\
& \left.+Q\left(i_{1}+i_{2}-i_{3}-i_{4}\right)\right] \\
= & D P\left(s_{4}-s_{3}\right)+2 D Q i_{\alpha} \\
= & D^{2} P \alpha+2 D Q i_{\alpha}, \\
J \ddot{\beta}= & D^{2} P \beta+2 D Q i_{\beta} .
\end{aligned}\right.
$$

\section{2 硬件实现及控制器设计}

闭环控制由一台主频 $133 \mathrm{MHz}$ 奔腾 $\mathrm{CPU}$ PC 机 实现数字控制, 在该 PC 机上运行的 3 个解耦控制器 分别对止推轴承的 3 个坐标轴进行控制, 使用 Advantech 公司的 16 位 A/D 卡 PCL-816 及 12 位 D/A 卡 PCL-726 进行模数和数模转换. 位移传感器模拟 量输出 $s_{1} \sim s_{4}$ 经 PCL-816 的 4 个独立通道转化为数字 量, 并送入控制闭环, 控制量 $i_{1} \sim i_{4}$ 经 PCL-726 的 4 
个独立通道, 转换为相应的模拟量信号, 由线性功率 放大器放大输出到驱动器线圈. 传感器零点设置在 传感器气隙为 $50 \mu \mathrm{m}$ 处.

平动 $\mathrm{z}$ 和 $\alpha, \beta$ 轴位移与驱动电流的关系可以从 (5)式推得:

$$
\left\{\begin{array}{l}
z=\frac{4 Q / M}{s^{2}-4 P / M} i_{z}, \\
\alpha=\frac{4 D Q / J}{s^{2}-D^{2} P / J} i_{\alpha}, \\
\beta=\frac{4 Q / J}{s^{2}-D^{2} P / J} i_{\beta} .
\end{array}\right.
$$

闭环控制使用带积分环节的控制器, 总体控制 框图如图 3 所示. 被控对象的参数, 对平动轴 $a_{0}=\frac{4 Q}{M}, b_{0}=\frac{4 P}{M}$, 对转动轴 $a_{0}=\frac{2 D Q}{J}, b_{0}=\frac{D^{2} P}{J}$, 闭环极点可以任意配置 ${ }^{[11,12]}$. 使闭环多重闭环极点 为 $-p$, 则控制器参数可由如下公式计算:

$$
\left\{\begin{array}{l}
A_{0}=4 p, \\
B_{1}=\left(6 p^{2}-a_{0}\right) / b_{0}, \\
C_{0}=p^{4} / b_{0} A_{0}, \\
B_{0}=\left(4 p^{3}-a_{0} A_{0}\right) / b_{0}-C_{0} .
\end{array}\right.
$$

控制器离散采用 Euler 后向差分法, 在 DOS 平台 上使用 $\mathrm{C}$ 语言编程, 采样时间为 $0.1 \mathrm{~ms}$.

\section{3 实验与讨论}

对主动空气轴承工作台的可控性进行了一部分 实验. 工作台的空气轴承供气系统, 由 $1.2 \mathrm{MPa}$ 往复 式压缩机, $0.25 \mathrm{~m}^{3}$ 储气罐, 和 $\mathrm{SMC}$ 气动清洁和压力调 节等部件构成. 压力调节采用精密稳压阀 IR2020-02BG. 系统没有供气时, 移动台自由地处于 基座上, 意外地观察到峰峰值为 $300 \mathrm{~nm}$ 传感器噪声. 噪声是由 4 个电涡流传感器信号调理电路的差频引 起的. 因此, 改用 4 个电容式位移传感器测量移动台 位移 ${ }^{[8]}$, 这 4 个电容式传感器分别封装在 2 个截面为 正方形的铜条中, 在基座上使用支架将这 2 个铜条非 接触置于移动台上方. 通过标定, 使电容式位移传感 器输出灵敏度与电浴流传感器一致.

当储气罐压力为 $0.8 \sim 1.0 \mathrm{MPa}$ 时, 使用稳压阀将 供气压力调节在 $0.8 \mathrm{MPa}$, 开环时移动台悬浮高度为 $30 \mu \mathrm{m}$ (气膜的厚度), 由于没有预载, 刚性和阻尼都 较差. 控制器的多重闭环极点设置在 $p=2 \pi \times 80 \mathrm{~Hz}$, 移动台通过电磁驱动器处于闭环控制时, 实现了可
以调节的预载, 静压气膜厚度为 $10 \mu \mathrm{m}$ (图 5). 当储气 罐压力低于 $0.8 \mathrm{MPa}$ 时, 移动台垂直位移稳定在 10 $\mu \mathrm{m}$ 处, 随着空气轴承的耗气, 供气压力不断下降, 驱动器的电流随着压力的下降而不断减小, 直至为 零. 图 6 是开环时, $1 \mathrm{~s}$ 内移动台垂直位移由于供气压 力不断下降从 $10 \mu \mathrm{m}$ 不断减小的情况. 从图 5 和图 6 中我们可以看到 $6 \mathrm{~nm} \mathrm{rms}$ 噪声, 这些噪声主要来自 传感器噪声. 由以上实验可知, 实现了可控的电磁预 载, 工作台的刚度和阻尼特性可控. 另外, 止推轴承 的 3 个轴也可以精确驱动.

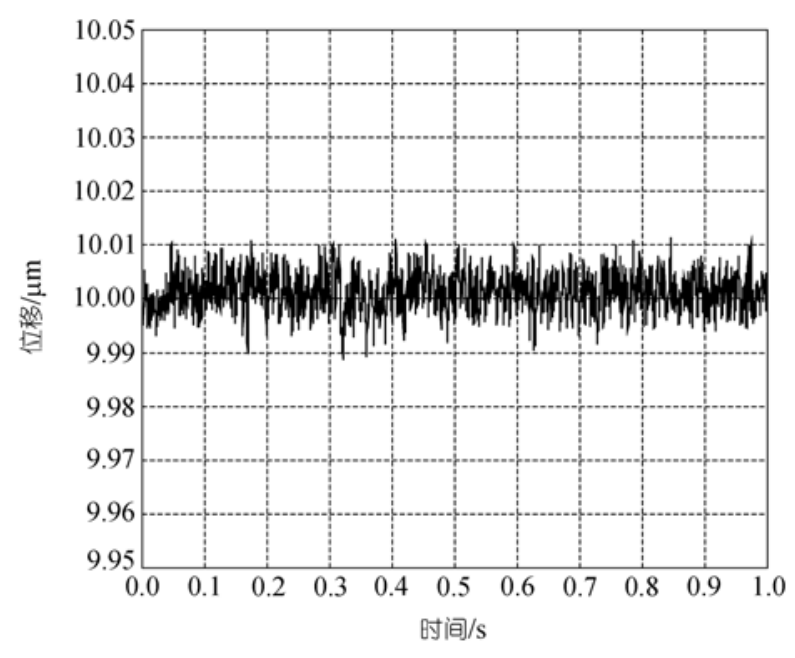

图 5 闭环极点 $p=80 \mathrm{~Hz}$ 时工作台气隙

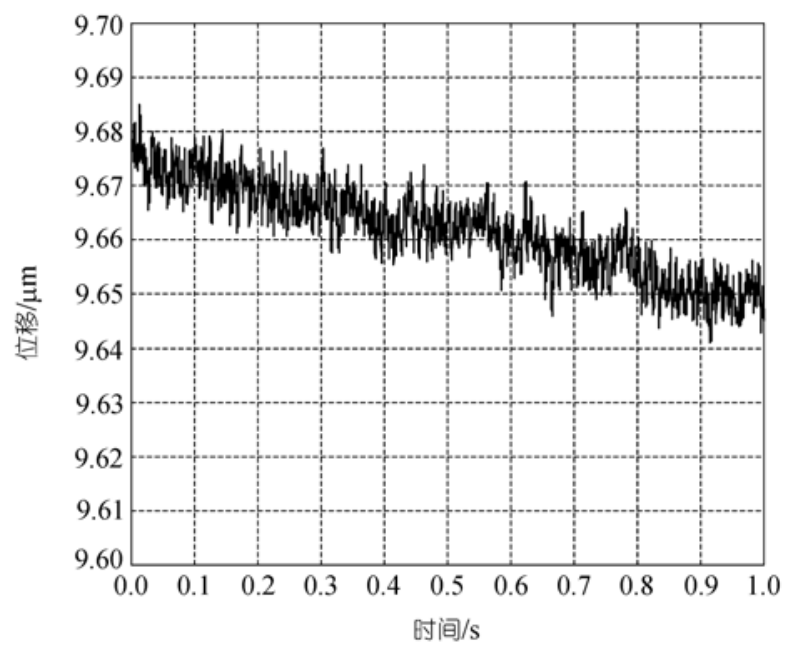

图 6 开环时工作台气隙

然而, 当主动空气轴承工作台按(5)式建模时, 忽略了静压空气轴承的静态和动态特性, 被控对象 
是开环不稳定的. 事实上, 被控对象在垂直方向上是 开环稳定的, 而且具有强烈的非线性. 因此, 在今后 的研究中, 需要计入静压空气轴承的影响来修正模 型, 以获得更好的闭环控制性能. 空气轴承对于止推 轴承转动坐标轴的影响可以忽略不计, 可以继续使 用(5)式的模型.

\section{4 结论}

实现了一个主动空气轴承工作台, 通过初步实 验证实了该工作台能够工作. 主动空气轴承工作台 使用了 4 个独立线圈构成电磁驱动, 电磁驱动器的铁 心直接加工在移动台轴承面上, 实现止推轴承 3 个解 耦的坐标轴的闭环控制, 采用一个气浮块实现了静
压空气悬浮. 实验表明, 主动空气轴承工作台工作时 的定位噪声 $\mathrm{rms}$ 值为 $6 \mathrm{~nm}$, 主要是由于传感器引起 的. 如果使用激光干涉仪位移测量装置, 能够有效地 降低传感器噪声, 达到更高的控制精度. 主动空气轴 承工作台具有结构简单, 性能不再取决于矩形静压 空气止推轴承, 无需被动预载等优点, 有可能取代矩 形静压空气止推轴承或磁悬浮轴承工作台用于一些 精密机床. 但是, 要达到纳米级位移测量分辨率, 需 考虑到电涡流传感器信号处理电路的同步. 此外, 在 主动空气轴承工作台具体应用以前, 还需要进一步 研究其电磁与静压空气混合力学特性模型, 以改善 闭环控制性能.

\section{参考文献}

1 Stout K J, Barrans S M. The design of aerostatic bearings for application to nanometer resolution manufacturing machine systems. Tribo Inter, 2000, 33: 803-809[DOI]

2 Kwan P Y B, Post B J. A tolerancing procedure for inherently compensated, rectangular aerostatic thrust bearings. Tribo Inter, 2000, 33: $581-585$ [DOI]

3 Slocum A, Basaran M, Cortesi R, et al. Linear motion carriage with aerostatic bearings preloaded by inclined iron core linear electric motor. Precis Eng, 2003, 27: 382-394 $\underline{\text { [DOI] }}$

4 Shinno H, Hashizume H, Yoshioka H, et al. $X$ - $Y$ - $\theta$ nano-positioning table system for a mother machine. CIRP, 2004, 53: 337-340

5 Peijnenburg A T A, Vermeulen J P M, Eijk van J. Magnetic levitation systems compared to conventional bearing systems. Microelectron Eng, 2006, 83: 1372-1375[DOI]

6 Jung K S, Baek Y S. Characteristics comparison of planar stages using repulsive and attractive type of surface actuator principle. Sensor Actuat A-Phys, 2005, 117: 173-182[DOI]

7 Kim W, Trumper D L. High-precision magnetic levitation stage for photolithography. Precis Eng, 1998, 22: 66-77[DOI]

8 李黎川, 丁王成, 卢秉恒. 高精度磁悬浮工作台及其解耦控制. 机械工程学报, 2004, 40(9): 84一88

9 Lee S Q, Gweon D G. A new 3-DOF Z-tilts micropositioning system using electromagnetic actuators and air bearings. Precis Eng, 2000, 24: 24-31묘]

10 Li L. Eddy-current displacement sensing using switching drive where baseband sensor output is readily available. IEEE Trans Instrum Meas, doi: 10.1109/TIM.2008.925004[DOD]

11 Mao J, Tachikawa H, Shimokohbe A. Precision positioning of a DC-motor-driven aerostatic slide system. Precis Eng, 2003, 27: $32-41 \underline{\text { DOI] }}$

12 Mao J, Tachikawa H, Shimokohbe A. Double-integrator control for precision positioning in the presence of friction. Precis Eng, 2003, 27: $419-428 \underline{\underline{\mathrm{DOI}]}}$ 